\title{
Patogeni isolabili da emocoltura
}

\section{Simona Roveta', Pierluigi Viale², Eugenio A. Debbia', Anna Marchese'}

\author{
' Sezione di Microbiologia - DISCAT, Università degli Studi di Genova \\ ${ }^{2}$ Clinica Malattie Infettive Tropicali, Università degli Studi di Brescia
}

L'emocoltura ha acquisito negli ultimi anni una sempre maggiore importanza, dovuta soprattutto all'aumento dell'incidenza di sepsi nei pazienti immunodepressi. Tuttavia, questo esame di laboratorio viene ancora richiesto in quantità relativamente scarsa rispetto alla reale rilevanza clinica (la sepsi è una delle più gravi sindromi infettive) e importanza diagnostica ed epidemiologica (quello che si isola dall'emocoltura è, nella grande maggioranza dei casi, il "vero" agente eziologico dell'infezione). Le setticemie rappresentano, dunque, una patologia importante, con una mortalità dal 5 al $60 \%$ a seconda si tratti di sepsi o shock settico, la tempestiva e corretta terapia antibiotica migliora significativamente la prognosi dei pazienti, fino al dimezzamento del tasso di letalità (9). Le principali situazioni cliniche in cui è importante eseguire un'emocultura sono: endocarditi ed infezioni endovascolari, polmonite batterica, pielonefrite ascendente, osteomielite ematogena, meningite batterica, ascessi endoaddominali, immunodepressioni di varia origine, cateterismi venosi e arteriosi, infezioni sistemiche ecc.

La terapia è inizialmente empirica, in attesa dei risultati del laboratorio che sono disponibili solamente dopo $24-48$ ore. Oltre a questa limitazione di carattere temporale vi sono altri fattori che occorre considerare quando si ricorre all'emocoltura per la diagnosi eziologica di una batteriemia: a) nessun mezzo di coltura è valido per tutti $\mathrm{i}$ potenziali microrganismi e alcuni a crescita lenta (es. micobatteri) richiedono un'incubazione prolungata; b) i falsi negativi sono piuttosto frequenti nei pazienti sottoposti a terapia antibiotica; i campioni di sangue dovrebbero essere raccolti prima della somministrazione di antibiotici, sebbene siano disponibili dei terreni di coltura contenenti sostanze che minimizzano gli effetti di questi farmaci sulla crescita batterica. c) l'interpretazione di una singola emocoltura positiva per alcuni microrganismi (es. CNS, corinebatteri e $P$. acnes) non è univoca $(14,10)$. Nei pazienti adulti dovrebbero essere raccolte due serie di emocolture per episodio febbrile per distinguere i probabili patogeni dai possibili contaminanti (5) specialmente quando occorre valutare la significatività di stafilococchi coagulasi negativi, corinebatteri, streptococchi viridandi e Bacillus spp. Solitamente è più facile trovare contaminanti in una singola serie di colture mentre i patogeni tipicamente si riscontrano da più di una serie di campioni (12). Una sola emocoltura positiva in assenza di sintomi o di segni clinici può riflettere una contaminazione, tuttavia qualsiasi emocoltura positiva merita un'attenta valutazione clinica prima di essere considerata come insignificante: questo vale anche nel caso di un risultato positivo per stafilococchi coagulasi negativi, spesso considerati in passato come contaminanti. Vi sono, inoltre, nella fase preanalitica molteplici elementi di fondamentale importanza da cui dipende il risultato ottimale dell'esame: le modalità di prelievo (la sede, il momento, la meccanica, il volume), il numero di campioni nelle 24 ore (1, 2, 3 o più) e l'intervallo tra l'uno e l'altro. I campioni di sangue dovrebbero essere raccolti appena prima del picco febbrile, ma generalmente vengono prelevati subito dopo l'innalzamento febbrile. Il volume totale di sangue raccolto è uno dei fattori più importanti per il ritrovamento del patogeno $(6,7)$ : in uno studio è stato riscontrato che aumentando da 40 a $60 \mathrm{ml}$ il volume del campione (raccolto in un periodo di 24 ore) si otteneva un $10 \%$ in più di campioni positivi (1). Esiste infatti una relazione diretta tra volume di sangue prelevato e positività: generalmente nell'adulto si preleva una quantità di circa $10 \mathrm{ml}$ di sangue per flacone (molti sistemi per emocoltura specificano il volume di sangue necessario per ottenere il risultato ottimale), mentre in età pediatrica, poiché la batteriemia presenta una carica microbica più elevata, di solito è sufficiente prelevare da $1 \mathrm{a} 5 \mathrm{ml}$ di sangue per flacone (8). Non dovrebbero essere raccolte più di quattro serie di campioni in un periodo di 24 ore; questo limite consente la valutazione di due episodi febbrili al giorno con due serie di emocolture per ogni episodio. Un numero maggiore di emocolture non è necessario, sulla base di dati provenienti da diversi studi è stato dimostrato che più del $95 \%$ delle batteriemie viene individuato con le prime 2 o 3 emocolture. Non bisogna dimenticare, infine, la possibilità di contaminazioni del campione al momento del prelievo con conseguenti difficoltà interpretative del risultato. Il significato dell'emocoltura dipende dunque da molteplici aspetti metodologici e interpretativi.

Le specie ricercate in routine sono generalmente 
costituite da un numero relativamente limitato, tuttavia modificazioni nella tipologia delle popolazioni microbiche (avvenute soprattutto in seguito all'incremento di soggetti immunocompromessi) e l'introduzione di approfondimenti diagnostici resi possibili dai progressi tecnologici hanno incrementato la frequenza e la tipologia degli isolamenti microbici dal circolo ematico.

Nell'ultimo decennio miglioramenti nelle tecnologie e nei terreni per le emocolture hanno portato alla realizzazione di sistemi automatizzati adatti all'individuazione di un'ampia gamma di microrganismi. L'isolamento di specie inusuali necessita di un'attenta valutazione ed interpretazione. L'isolamento può derivare, ad es., da una batteriemia a carattere transitorio che può coinvolgere anche un numero cospicuo di specie abitualmente endogene $\mathrm{o}$ ambientali. La positività di un solo campione e la negatività degli altri rappresentano validi criteri di discriminazione per considerare, entro certi limiti, un reperto di questo tipo "fisiologico", tuttavia un reperto positivo in un soggetto "a rischio" necessita comunque di un'indagine più approfondita.

Tenendo conto delle suddette modificazioni epidemiologiche occorre usare prudenza nell'interpretare il significato di quei microrganismi un tempo catalogati come "contaminanti". Attualmente viene dedicata attenzione alle numerose specie di Corynebacterium (particolarmente a quei gruppi indicati un tempo come "difterici non tossici") e Bacillus, mentre nel passato la loro presenza era attribuita a disinfezione non corretta o ad asepsi nel prelievo del campione. Specie batteriche ospiti dell'uomo o degli animali (o anche tipicamente ambientali) possono occasionalmente essere rinvenute nel torrente circolatorio e correlate a una patologia, specialmente in soggetti che presentano compromissione del sistema immunitario (3).

Come già accennato, diversi batteri in grado di causare setticemia non vengono rilevati dalle procedure di routine per le emocolture. Leptospira, ad esempio, non viene evidenziata con i terreni di coltura standard (13). Brucella può crescere in un comune terreno per emocoltura, ma occorre conservare la coltura per tre settimane e spesso è anche necessaria una subcultura finale $(2,15)$. Particolare considerazione viene attualmente rivolta ad alcuni generi esigenti o non usuali, quali Bartonella, Mycoplasma, Legionella, Coxiella, Abiotrophia, Listeria, Chlamydia. Oggetto di interesse è anche un gruppo di microrganismi (geneticamente non correlati tra loro), culturalmente esigenti e non evidenziabili dalle pratiche routinarie, indicati come HACEK (Haemophilus aphrophilus, Actinobacillus acti- nomycetemcomitans, Cardiobacterium hominis, Eikenella corrodens, Kingella kingae), si tratta di ospiti abituali del cavo oro-faringeo che possono essere associati a endocarditi batteriche. I microrganismi di questo gruppo possono essere evidenziati tramite i terreni di coltura utilizzati in routine, ma necessitano di 14 giorni di incubazione e può essere necessario eseguire una subcultura finale.

Occorre quindi considerare l'eventualità che una aliquota dei batteri isolati (gli "inusuali" non previsti nella normale routine) non venga tenuta nella giusta considerazione e che vi siano false negatività dovute a particolari esigenze nutrizionali od alla impossibilità di coltivazione in vitro.

L'incidenza di questa aliquota appare tutt'altro che trascurabile e caratterizza quei quadri patologici (es. endocarditi) indicati comunemente come "sindromi da emocoltura negativa".

L'emocoltura per micobatteri è divenuta sempre più richiesta in seguito al dilagare dell'HIV negli anni '90, inizialmente veniva isolato pressoché esclusivamente il bacillo tubercolare, in seguito si sono aggiunti anche $M$. avium (negli USA si era già diffuso quando ha cominciato a essere rinvenuto anche in Italia) e $M$. genavense (gli isolamenti italiani sono stati tra i primi). L'aumentata richiesta ha portato allo sviluppo di terreni e apparecchiature sempre più sensibili rivelando l'inadeguatezza dell'impiego di terreni solidi se non abbinati alla procedura della lisi-centrifugazione. Le apparecchiature automatiche utilizzano un sistema basato sulla produzione di $\mathrm{CO}_{2}$ (radiometria) oppure sul consumo di $\mathrm{O}_{2}$ (fluorescenza, variazione di pressione). Quando l'introduzione di terapie adeguate ha ridotto il rischio di infezioni batteriche nei pazienti con HIV, il numero di micobatteriosi disseminate si è ridotto molto notevolmente. Pertanto la richiesta di ricerca colturale di micobatteri nel sangue si è ridotta di circa il 70\% negli ultimi 5 anni (11).

Tra i microrganismi isolati da emocolture la Candida, che rappresenta dall'8 al 15\% delle infezioni nosocomiali, si posiziona al quarto posto. Quando si sospetta la presenza di un micete, l'analisi microbiologica tradizionale necessita di alcune precauzioni operative inerenti la modalità di incubazione: la temperatura deve essere di $35^{\circ} \mathrm{C}$, i flaconi devono essere agitati per favorire l'aerobiosi e l'incubazione deve avere una durata di almeno 7 giorni. L'inoculo di volumi di sangue superiore rispetto a quanto richiesto per i batteri può migliorare la sensibilità diagnostica. L'emocoltura effettuata con il metodo di lisi-centrifugazione è altamente sensibile e riduce i tempi di incubazione. Vi sono poi altre considerazioni inerenti la coltura dei miceti dal sangue: sebbene 
i lieviti si sviluppino in terreno liquido entro 7296 ore di incubazione, alcuni come $C$. neoformans e $H$. capsulatum hanno dei tempi più lunghi di crescita (10 e 30 giorni rispettivamente) (4). Un'alternativa all'emocoltura nella diagnosi di batteriemia è rappresentata dalle metodiche molecolari basate sulla PCR: queste metodiche forniscono i risultati con una rapidità molto maggiore e presentano anche una sensibilità più elevata (soprattutto nei pazienti precedentemente trattati con antibiotici o con infezioni da microrganismi difficili da coltivare) tuttavia la specificità non è elevatissima e i costi sono maggiori rispetto a quelli dell'esame tradizionale.

\section{BIBLIOGRAFIA}

1. Auckenthaler R, Ilstrup DM, Washington JA. Comparison of recovery of organisms from blood cultures diluted 10\% (volume/volume) and 20\% (volume/volume). J Clin Microbiol 1982; 15: 860-4.

2. Bannatyne RM, Jackson MC, Memish S. Rapid diagnosis of Brucella Bacteremia by using the BACTEC 9240 system. J Clin Microbiol 1997; 35: 2673-4.

3. Casolari C, Fabio A, Fabio U. Incidenza e frequenza nel sangue di batteri "inusuali " od "esigenti". Microbiologia Medica 2002; 17: 72-3.

4. Farina C. "I miceti". Microbiologia medica 2002; 17: 74.

5. Kellog JA, Ferrentino FL, Liss J, Shapiro SL, Bankert DA. Justification and implementation of a policy requiring two blood cultures when one is ordered. Lab Med 1994; 25: 323-30.

6. Li J, Plorde JJ, Carlson LG. Effects of volume and periodicity on blood cultures. J Clin Microbiol 1994; 32: 2829-31.

7. Mernel LA, Maki DG. Detection of bacteremia in adults: consequences of culturing on inadequate volume of blood. Ann Intern Med 1993; 119: 270-2.

8. Paisley JW, Lauer BA. Pediatric blood cultures. Clin Lab Med 1994; 14: 17-30.

9. Paradisi F, Corti G, Sbaragli S. Il punto di vista dell'infettivologo. Microbiologia Medica 2002; 17: 71-2.

10. Reimer LG, Wilson ML, Weinstein MP. Update on detection of bacteremia and fungemia. Clin Microbiol Rev 1997; 10: 444-65.

11. Tortoli E. La ricerca colturale di micobatteri nel sangue. Microbiologia Medica 2002; 17: 73.

12. Weinstein MP, Reller LB, Murphy JR, Lichtenstein KA. The clinical significance of positive blood cultures: a comprehensive analysis of 500 episodes of bacteremia and fungemia in adults. I. Laboratory and epidemiologic observations. Rev Infect Dis 1983; 5: 35-53.

13. Weyant RS, Bragg SL, Kaufmann AF. Leptospira and Leptonema. In: Manual of Clinical Microbiology $7^{\text {th }}$ ed, American Society for Microbiology, Washington DC 1999; 739-45.

14. Wilson ML, Weinstein MP. General principles in the laboratory detection of bacteremia and fungemia. Clin Lab Med 1994, 14: 69-82.

15. Yagupsky P, Peled N, Press J, Abramson O, Aburashid M. Comparison of BACTEC 9240 peds plus medium and Isolator 1.5 microbial tube for detection of
Brucella melitensis from blood culture. J Clin Microbiol 1997; 35: 1382-84.

\section{Eugenio A. Debbia}

Università degli Studi di Genova,

Sez. Microbiologia-DISCAT

Largo Rosanna Benzi 10,

16132 Genova - Italia

Tel ++39 010-3537655, Mobile 3292605218

Fax ++39010504837

Direct fax ++390103537648

E-mail: eugenio.debbia@unige.it 